\title{
EVALUACIÓN Y METODOLOGÍAS DOCENTES Y SU INCIDENCIA EN LAS COMPETENCIAS GENÉRICAS: PERSPECTIVAS TEÓRICAS
}

\author{
Freddy Marín-González \\ Universidad de la Costa (Colombia) \\ fmarin1@cuc.edu.co \\ Jesús N García \\ Universidad de León/Universidad de la Costa, \\ Alicia Inciarte \\ Universidad de la Costa (Colombia) \\ Edgardo Sánchez \\ Universidad de la Costa (Colombia) \\ Marcial Conde \\ Universidad de la Costa (Colombia) \\ Judit García-Martín \\ Universidad de Salamanca
}

Fecha de Recepción: 9 Abril 2019

Fecha de Admisión: 30 Abril 2019

\section{RESUMEN}

El artículo tiene como propósito aproximarse a la construcción de un marco teórico de sustento que permita comprender la interrelación entre las variables identificadas en el estudio. Específicamente, la construcción teórica atiende a cinco perspectivas de análisis, a saber: enseñanza centrada en competencias y el EEES; metodologías activas universitarias hacia una web ubicua 4.0; la dimensión instruccional psicológica y curricular; tecnología de validación de instrumentos y la validación de intervenciones basadas científicamente desde indicadores empíricos (IBE). Este proyecto pretende estudiar las metodologías docentes y las formas de evaluación transversales de las asignaturas, con énfasis en las competencias genéricas de lectura crítica y construcción textual; se prevé el análisis de las prácticas docentes y las necesidades de formación en el estudiante, como referente para la concepción de estrategias eficientes que contribuyan a mejorar el proceso enseñanza-aprendizaje. El enfoque metodológico está centrado en procesos de revisión y análisis sistemáticos, que permitan la profundización teórica que den cuentan de los diversos constructos teóricos, con el foco en generar un sistema relacional de categorías y enfoques teóricos que aporten luz tanto para la construcción de instrumentos de evaluación como estrategias de actuación y contribuyan en la mejora docente y en la calidad de la enseñanza y repercutan en mejores resultados académicos en los estudiantes universitarios en las pruebas de competencias genéricas. Se 


\section{EVALUACIÓN Y METODOLOGÍAS DOCENTES Y SU INCIDENCIA EN LAS COMPETENCIAS GENÉRICAS: PERSPECTIVAS TEÓRICAS}

concluye con la necesidad de un marco teórico en torno a cinco ejes que guía y orienta el proceso de investigación, de interpretación de datos y evidencias y el protocolo de actuación y propuestas de mejora de la calidad docente. Se espera aportar luces no solo para la aplicación y mejora docente, sino también para las implicaciones teóricas que se desprenden de los mismos, y permita una adecuada articulación de los diferentes constructos científicos medidos y para dar sentido a las lagunas actuales en el conocimiento científico sobre el tema. "Proyecto de Investigación Competitivo CONV-ÍNDEX Núm. 13-2018, Código Proyecto INV. 150-01-007-13 Universidad de la Costa (Colombia) - Universidad de León (España). Evaluado por la ACAC (MEN Colombia)"

Palabras clave: competencias; psicología instrucción; tecnología; validación instrumentos; intervenciones basadas evidencia (IBE)

\section{ABSTRACT}

Evaluation and teaching methodologies and their incident in generic competences: theoretical perspectives. The purpose of the paper is to approximate the construction of a theoretical framework of sustenance that allows understanding the interrelation between the variables identified in the study. Specifically, the theoretical construction covers five perspectives of analysis, namely: competency-focused teaching and the EHEA; active university methodologies towards a ubiquitous web 4.0; the instructional, psychological and curricular dimension; instrument validation technology and the validation of scientifically based interventions based on empirical indicators (IBE). This research project aims to study the teaching methodologies and the transversal evaluation forms of the subjects, with emphasis on the generic competences of critical reading and textual construction; the analysis of the teaching practices and the training needs of the student is foreseen, as a reference for the conception of efficient strategies that contribute to improve the teaching-learning process. The methodological approach is focused on systematic review and analysis processes, which allow the theoretical deepening of the various theoretical constructs, with the focus on generating a relational system of categories and theoretical approaches that provide light for the construction of instruments evaluation as strategies of action and contribute in the improvement of teaching and in the quality of education and impact on better academic results in university students in generic competency tests. It concludes with the need for a theoretical framework around five axes that guides and guides the process of research, interpretation of data and evidence and the protocol of action and proposals for improving the quality of teaching. It is expected to provide lights not only for the application and teacher improvement, but also for the theoretical implications that arise from them, and allow an adequate articulation of the different scientific constructs measured and to give meaning to the current gaps in scientific knowledge about the topic. "Competitive Research Project CONV-ÍNDEX No. 13-2018, Project Code INV. 150-01-007-13 University of the Coast (Colombia) University of León (Spain). Evaluated by the ACAC (MEN Colombia)"

Keywords: competencies; instructional psychology; technology validation instruments; evidence based interventions (EBI)

\section{INTRODUCCIÓN}

Como parte del proyecto de investigación "Evaluación y Metodologías e Incidencias en las Competencias Genéricas (EMICOG)" centrado en el estudio de las metodologías docentes y las formas de evaluación transversales de las asignaturas, con énfasis en las competencias genéricas de lectura crítica y construcción textual, es necesaria la construcción de un marco teórico de sustento para las principales variables trabajadas en el estudio. La información recogida en el marco teórico proporciona un conocimiento de la teoría que da sustento a la investigación, como referente para avanzar en la producción de nuevo conocimiento. 
La idea básica es implementar un programa de investigación centrado en la innovación docente universitaria, abordada de forma integrada desde diferentes perspectivas, con el objetivo de ser relevante curricular y socialmente, además de aportar conocimiento nuevo disponible para la toma de decisiones en cuanto a la mejora del proceso de evaluación y de las metodologías docentes utilizadas por los profesores y que contribuirán, previsiblemente, en mejores resultados en competencias genéricas en los estudiantes.

La concepción del referido proyecto ubicado en el contexto de la educación superior, orienta su discusión y análisis a cómo desde la gestión del currículo en programas académicos se conciben estrategias para el desarrollo de competencias, específicamente en los procesos de mediación didáctica y evaluación de los aprendizajes, de forma tal que redunden en un mejor rendimiento académico.

En lo metodológico está centrado en procesos de revisión y análisis sistemático. De modo que la argumentación y profundización de cuenta de los diversos constructos, con el propósito de generar un sistema relacional de categorías y enfoques teóricos, para fundamentar la construcción de instrumentos de evaluación y de estrategias de actuación que contribuyan a la mejora de la función docente, así como el afianzamiento de los aprendizajes, a través de un incremento significativo en la calidad educativa que se evidencie en mejores resultados en pruebas externas sobre competencias genéricas de los universitarios.

Por consiguiente, el marco teórico del referido proyecto articula de forma integral y sistémica cinco ejes o perspectivas nucleares: 1) enseñanza centrada en competencias y el EEES (Espacio Europeo de Enseñanza Superior); 2) metodologías activas universitarias hacía una web ubicua 4.0; 3) la perspectiva instruccional psicológica y curricular; 4) tecnología de validación de instrumentos; 5) y por último la validación de intervenciones basadas científicamente bajo las pautas de indicadores basados empíricamente (IBE).

\section{MARCO TEÓRICO DESDE CINCO PERSPECTIVAS}

\section{La enseñanza centrada en competencias y el EEES (Espacio Europeo de Enseñanza Superior).}

La perspectiva de armonización y homologación en el Espacio Europeo de Educación Superior que ha centrado su foco en una formación superior en competencias (CUC, 2018; Fidalgo \& García, 2008), que trata de promover personas altamente formadas de modo integral, flexible, y adaptable para las exigencias muy cambiantes y competitivas del mundo globalizado actual y de una sociedad del conocimiento. Está claro que hay un gran debate sobre los límites de la valoración de competencias (Barnet, 2001), sobre la complejidad de los procesos valorativos que se concretan en sistemas de evaluación (Díaz-Flórez, 2018), en el contexto de la educación superior en Colombia (OCDE, 2016), con una necesidad clara de mejora de calidad de enseñanza (Tamayo et al., 2018) sobre todo en relación con la formación de docentes en Colombia (Arias-Gómez et al., 2018) y de las paradojas actuales en educación y que afectan a las cuestiones abordadas en esta investigación (Ravitch, 2013), por lo que se considera una visión integral y comprehensiva de valoración de la enseñanza y del aprendizaje y del constructo competencia genérica comunicativa, de lectura crítica y construcción textual.

Esta dimensión se fundamenta teóricamente en los aportes de la Organización para la Cooperación y Desarrollo Económico (OCDE - 2016), al definir una estrategia con la finalidad de contribuir a que los países conciban políticas de desarrollo de mayor pertinencia para fortalecer la generación de empleos. Se plantea la necesidad del aprendizaje entre pares y el fortalecimiento de relaciones intergubernamentales. Específicamente los informes del Programa para la Evaluación 


\section{EVALUACIÓN Y METODOLOGÍAS DOCENTES Y SU INCIDENCIA EN LAS COMPETENCIAS GENÉRICAS: PERSPECTIVAS TEÓRICAS}

Internacional de Alumnos (PISA) cuyo análisis arrojan datos importantes en cuanto a las habilidades que desarrollan los estudiantes en diferentes disciplinas científicas.

Para la OCDE la noción de competencia tiene una amplia dimensionalidad, trasciende la propia acepción de habilidad o capacidad; se trata en definitiva de fortalecer condiciones para responder de manera ágil y efectiva a las transformaciones del contexto; ser consciente de las demandas de situaciones complejas que requieren habilidades, actitudes, y recursos que contribuyan a que los individuos alcancen grados importantes de desarrollo en el plano personal y profesional.

Los autores coinciden en la polisemia conceptual del término competencia, denotan una evolución en la acepción tradicional para asociarlo a procesos de complejidad creciente, en atención a los grados de desarrollo que alcanza un individuo. Algunos destacan la necesidad de fortalecer los perfiles de desempeño desde las capacidades que tiene el sujeto para actuar en contextos bien definidos y para participar propositivamente en su transformación (Marín et al, 2018). La formación por competencias no solo atiende los requerimientos de las disciplinas, profesiones y contextos, sino que se ubica en el plano formativo del sujeto en un componente intrapersonal e interpersonal, lo cognitivo, destrezas, habilidades, valores, actitudes. En este sentido, se definen competencias genéricas comunes a la formación en las distintas opciones curriculares y las competencias específicas que determinan la identidad de cada profesión u oficio.

\section{Metodologías activas universitarias hacía una web ubicua 4.0}

En segundo lugar, el gran desarrollo de la implementación de metodologías activas, en parte producto del enfoque de competencias promovido en Europa desde el EEES; en parte, como desarrollo de la perspectiva instruccional; en parte, como agotamiento frente a las metodologías tradicionales ineficientes ante los nuevos retos de la sociedad del conocimiento y del paso desde la web 1.0 (internet básico), por la web 2.0 (red social, la de la colaboración), a la web 3.0 (red semántica, metadatos semánticos), y ser competentes en la web 4.0 (web móvil, web ubicua) y dar respuestas sociales más sostenibles, satisfactorias y eficientes. La ilustración de las modalidades de eLearning, 0 del aprendizaje basado en problemas (ABP, García, 2002; 2014; 2016) son una evidencia clara al respecto de la versatilidad y de las potencialidades que ofrecen en la conquista de una formación profesional avanzada y altamente funcional y flexible que permita dar respuestas satisfactorias a las necesidades y retos sociales.

En este contexto, la Organización para el Desarrollo Económico (OCDE), evidencia inquietudes en correspondencia con los indicadores de desempeño de los estudiantes de los diferentes niveles educativos en pruebas externas. En el caso del Programa Internacional de Evaluación de Estudiantes (PISA), es reiterativa la preocupación para los países latinoamericanos; en el caso de Colombia, desde el año 2006, cuando participa por primera vez en esta medición, los resultados siguen siendo preocupantes. Ello ha derivado en la concepción de políticas educativas que no solo atiendan a problemas de cobertura, sino que trasciendan al ámbito de las prácticas educativas y la mediación didáctica-pedagógica, con su correspondiente correlato en el desarrollo de competencias.

Específicamente se orienta la concepción a evaluar competencias que desarrollan los estudiantes, asociadas a los dominios cognitivo, procedimental y actitudinal en el componente abstracto y empírico de las diferentes disciplinas científicas; tal es el caso del lenguaje, y más concretamente los procesos de escritura y comprensión lectora. Desde los aportes de autores como Cantoral (2014), se hace necesario que procesos como la mediación didáctica y la práctica pedagógica sean de corte constructivista y orientadoras del cambio conceptual. Por tanto, las pruebas externas permiten la validación de las competencias desarrolladas en las diferentes áreas; se asume una perspectiva integradora en el sentido de que las competencias aun cuando están nucleadas desde los 
saberes propios de cada área, el fortalecimiento de capacidades se fundamenta en la convergencia interdisciplinar. Para el área de lenguaje, específicamente en cuanto a competencias escriturales y de comprensión lectora, los estudiantes evidencian debilidades referidas a la argumentación, identificación de ideas principales y secundarias, secuencialidad, lógica y coherencia; estas falencias, propias de la expresión oral o escrita, afectan de la misma forma a las diferentes áreas del currícu10.

En la búsqueda de estrategias para fortalecer el perfil por competencias del estudiante, incluido el de educación superior, se han definido políticas de gestión que viabilicen la integración de las tecnologías de la información y la comunicación en la mediación didáctica-pedagógica. Desde los aportes de Améstica et al (2014); Marín et al (2017), Sunkel et al (2013) explican la importancia de la informatización escolar como un proceso donde el currículo se construye en el plano de las interacciones, entre actores docentes-estudiantes, con la disposición de unidades y recursos organizacionales de carácter tecnológico.

La inclusión en la mediación didáctica de tecnologías de apoyo para el fortalecimiento de competencias genéricas está fundamentada en procesos de interacción e intercambio en torno a los sistemas de aprendizaje. De Castro (2012) destaca la necesidad de la creación de dispositivos cotidianos que evidencien experiencias coherentes derivadas del mundo real. Se hace necesaria la integración de tecnologías de apoyo con interfaces amigables de manera que fluya la interacción entre los usuarios y los dispositivos. De allí que se plantea como una estrategia válida para orientar el desarrollo de competencias genéricas en comprensión lectora, la integración de metodologías activas universitarias hacía una web ubicua 4.0; las tendencias emergentes en este sentido refieren sistemas de aprendizaje inteligentes de naturaleza ubicua cuyo principal soporte está representado por internet y las múltiples posibilidades que trae su apropiación por parte de los estudiantes.

\section{Perspectiva instruccional psicológica y curricular}

En tercer lugar, la perspectiva instruccional, tanto desde la psicología como desde los estudios didácticos y pedagógicos (curriculares y educativos) que han permitido desarrollar enfoques estratégicos y de procesos, no solo de productos, de comprensión del dominio y adquisición de competencias en relación con variables determinantes, mediadoras o moduladoras como autoeficacia percibida, motivación y estrategias de aprendizaje y afrontamiento, estilos de aprendizaje y pensamiento, atribuciones de causa de éxitos y fracasos, variables emocionales y sociales, o en relación con el establecimiento de metas académicas y de logro diversas; incluyendo otro tipo de mejoras en la promoción de condiciones óptimas para el aprendizaje.

El proyecto de Evaluación y Metodologías e Incidencias en las Competencias Genéricas (EMICOG) orientado a estudiar las metodologías docentes y las formas de evaluación transversales de las asignaturas, con énfasis en las competencias genéricas de lectura crítica y construcción textual, tiene un referente de base interdisciplinar. La construcción del conocimiento sobre las competencias genéricas y las metodologías de enseñanza es un proceso de grandes implicaciones, que no puede ser explicado desde una única perspectiva. Al respecto convergen dominios científicos para explicar los procesos instruccionales que están asociados tanto a la psicología como al currículo.

El proceso instruccional como base para el desarrollo de competencias genéricas se orienta hacía la concepción de una aprendizaje significativo; para ello es necesario estudiar las interrelaciones entre los hechos y variables que forman parte del plano de la realidad; se requiere un abordaje metodológico y epistemológico para comprender las diferentes vías a través de las cuáles se produce el conocimiento sobre el objeto de estudio. Para García (2011) la construcción de un conocimiento de base interdisciplinar representa un sistema complejo que integra la observación, el regis- 


\section{EVALUACIÓN Y METODOLOGÍAS DOCENTES Y SU INCIDENCIA EN LAS COMPETENCIAS GENÉRICAS: PERSPECTIVAS TEÓRICAS}

tro de datos y la organización de procesos e implica en el individuo procesos de desarrollo cognitivo y metacognitivo.

La construcción del conocimiento como producto de la gestión didáctica y curricular implica acciones intencionales del sujeto, quien se hace consciente de la necesidad de participar en la transformación de la realidad, como base de lo que debe ser el avance de la ciencia. Desde una perspectiva instruccional el desarrollo de competencias como proceso inherente al sujeto que aprende, representa una acción intencional; dado que se elaboran representaciones mentales que implican abstracción, sistematización, organización mental de las ideas y el conferir significados. Al respecto, Padrón (1998), destaca que el proceso de cognición asociado al desarrollo de competencias, supone la generación de conceptos empíricos sobre realidad preexistente y conceptos teóricos que parten de la relación saber/hacer; teoría/praxis, competencias/ejecución.

\section{Tecnología de validación de instrumentos}

En cuarto lugar, la perspectiva renovada y los avances de la tecnología de validación de instrumentos de evaluación, tanto en cuanto al gran desarrollo psicométrico del cálculo de validez y fiabilidad, como en cuanto a su utilización y control por medio de aplicaciones online sofisticadas y eficientes (ej. SurveyMonkey), con un gran ahorro material (no hace falta soporte físico) como en cuanto a la población a la que pueden llegar los muestreos 0 el momento en que pueden cumplimentarlo. Una ilustración de ello son las publicaciones recientes a nivel internacional, punteras en el campo, Educational Psychology, y análisis de datos con técnicas rigurosas como el análisis de ítems, el análisis factorial exploratorio (AFE) y el análisis factorial confirmatorio (AFC), junto con el uso de sistemas de ecuaciones estructurales (SEM) para el análisis de validación de instrumentos (Batista, Coenders \& Alonso, 2004; Cheng \& Lin, 2018; Cuesta, Suárez, Lozano, García-Cueto \& Muñiz, 2018; Fang, Zhang, Liu, Pan, Jin \& Nie, 2017; Fuente, Cubero, Sánchez, Peralta, Garzón \& Fiz, 2017; Gil, Cecato \& Marí, 2018; Habok \& Magyar, 2018; Lai, Zhao \& Cheng, 2018; MartínezFerrer, Moreno \& Ochoa, 2018; Milienos, Karagiannopoulou \& Athanasopoulos, 2018; Nie, Teng, Zhang Pany, 2017; Oriol, Miranda, Oyanwedel \& Torres, 2017; Pérez \& Medrano, 2010; Peris, Maganto \& Garaigordobil, 2018; Puente, Páez, Ubillos \& de Costa, 2018; Rey, Mnez \& Calonge, 2018; Romera, Herrera, Casas, Ortega \& del Rey, 2018; Sinval, Pinto, Queirós \& Maroco, 2018; Taasoobshirazi \& Wang, 2016; Tian \& Gao, 2018; Ventura, 2018; Ventura, Barboza \& Caycho, 2018; Ventura \& Caycho, 2017; Ventura, Caycho, Vargas \& Flóres, 2018; Widlund, Tuominen \& Korhonen, 2017; Zen et al., 2019). En esta línea, se pretende avanzar en la validación de instrumentos desde las investigaciones propias desarrolladas recientemente por nosotros (Álvarez \& García, 2014; 2015; Canedo, García \& Pacheco, 2017; Díaz \& García, 2016; 2017; García-Martín \& García-Sánchez, 2013; 2015; 2016; 2017; 2018; García-Martín et al, 2014; Robledo \& García, 2018).

La validación de instrumentos resulta clave en cualquier proceso de naturaleza didáctica o de investigación. En el proyecto de Evaluación y Metodologías e Incidencias en las Competencias Genéricas (EMICOG) orientado a estudiar las metodologías docentes y las formas de evaluación transversales de las asignaturas, desde su carácter riguroso y científico se hace necesario el diseño, validación y aplicación de instrumentos que de forma estandarizada permitan la recolección apropiada de la información.

\section{Perspectiva de las intervenciones basadas empíricamente}

Y en quinto, la validación de actuaciones pedagógicas, didácticas e instruccionales siguiendo los indicadores basados empíricamente 0 científicamente. Estos indicadores, controles de calidad y niveles de evidencia científica se han venido desarrollando con enfoques muy diversos, desde el 
ámbito médico y de la salud, pasando por el psicológico, el educativo-pedagógico-didáctico-instruccional, el profesional respectivo, el de la gestión política de salud y el de la educación entre otros. Un aspecto esencial es el de la fidelidad de los tratamientos basados en indicadores IBE (García, 2014, 2016; et al., 2016). Estos indicadores son esenciales para los estudios que se desarrollen en la segunda fase del proyecto de investigación en que se validen prácticas exitosas en desarrollo y se implementen nuevas estrategias transversales de fomento de las competencias genéricas en todas las asignaturas de la Universidad. Se han implementado muchas directrices y acciones educativas basados en las mejores prácticas (USOE, 2019), o centrado en configuraciones de innovación y toma de decisiones centrado en prácticas basadas en la evidencia (CEEDAR, 2019a, b).

\section{DISCUSIÓN Y CONCLUSIONES}

La enseñanza y el aprendizaje centrado en competencias, mediante el uso de metodologías docentes activas y dentro de una web ubicua (web 4.0), la introducción de la tecnología de diseño, la aplicación y validación de instrumentos, junto con los avances y controles de calidad (indicadores $\mathrm{IBE}$ ), son el marco instruccional en el que se inscribe este programa de investigación a cuatro años. Este se lleva a cabo en dos fases sucesivas: i) detección de necesidades e ii) implementación de estrategias transversales, que se van solapando para logro de las metas propuestas.

El estudio de la valoración integral de los procesos de enseñanza y aprendizaje se realiza mediante sistemas de evaluación diversos y comprehensivos, que permiten la detección de situación y necesidades; siendo dicha información la base para la toma de decisiones didácticas e instruccionales en la práctica cotidiana en las disciplinas. En este sentido, la mejora de la adecuación, eficacia y calidad docentes se sitúan en una encrucijada compleja de la evaluación (Tamayo et al., 2018), incluida la educación superior en Colombia (OCDE, 2016), dados los límites de lo evaluable, como las competencias (Barnet, 2001) y de la simplificación que pudiera suponer abordar la evaluación sin considerar el contexto educativo, didáctico, social, siendo más importante la calidad de la enseñanza que los propios sistemas de evaluación (Ravitch, 2013). Igualmente, el debate sobre las competencias en educación superior es de gran interés y supone considerar diversas alternativas (Díaz-Flórez, 2018) de forma específica, también, en el contexto cultural, pedagógico y de las necesidades sociales de desigualdad de Colombia, máxime en la formación específica de profesores (Arias-Gómez et al., 2018; García-Martín \& García-Sánchez, 2017; Scott et al., 2018).

En este debate, se focalizan las posibilidades reales de una investigación, que no resolverá las desigualdades ni necesidades de reforma integral pero que contribuirá en la profesionalización de los docentes, en la mejora de la valoración comprehensiva de la enseñanza y aprendizaje, y en la consecución de mejores resultados en las competencias genéricas de comunicación en los estudiantes en el contexto de las diferentes disciplinas.

La cuestión clave que persiste en este debate es la de obtener evidencias empíricas validadas, más allá de las propias reflexiones que pueden guiar el proceso de investigación. Esta ausencia de evidencias empíricas contrastadas, es un argumento añadido para este estudio.

La revisión sistemática reciente literacy across disciplines en profesores en formación de Scott et al (2018), es paradigmática. De 3413 estudios identificados en los últimos cincuenta años en USA, solo cumplieron los criterios de inclusión 29 , de los cuales solo tres lo fueron cuasi-experimentales (con un grupo de control y medidas pre-post) y los otros 26 estudios de observación de experiencias de calidad, ni siquiera cuasi-experimentales (con una única medida). Lo mismo sucede con la revisión del aprendizaje auto-regulado y su papel en el rendimiento académico en los últimos veinte años en China (Li et al., 2018), quienes, de 603 estudios encontrados, solo pudieron incluir 59 que cumplieran criterios mínimos de calidad, y de ellos, solo 4 cuasi-experimentales y 55 


\section{EVALUACIÓN Y METODOLOGÍAS DOCENTES Y SU INCIDENCIA EN LAS COMPETENCIAS GENÉRICAS: PERSPECTIVAS TEÓRICAS}

observacionales con una única medida (Li et al., 2018).

Ello muestra que la base de antecedentes de la que se parte es sobre todo conceptual, basada en experiencias impresionísticas, carente de estudios de validación que cumplan los estándares de calidad y rigor para poder hacer generalizaciones. La necesidad de enfocar objetivamente la valoración, las metodologías activas y los resultados en competencias genéricas comunicativas parece obligada. En este camino, las reflexiones y conceptualizaciones son de interés, pero se precisan modelos didácticos y pedagógicos concretos, como los que se desprenden de la revisión de estudios de los últimos cincuenta años de literacy across disciplines de Scott et al., (2018), o como se muestra en el camino que está siguiendo la competencia genérica comunicativa de construcción textual como actividad de aprendizaje 0 writing as a learning activity (Klein \& Boscolo, 2016), o como se sugiere de la revisión del aprendizaje auto-regulado y su papel en el rendimiento académico en los últimos veinte años en China (Li et al., 2018), por citar tres grandes ámbitos culturales (USA, Europa, China). Sin embargo, en Colombia no se ha encontrado ningún estudio de revisión de estudios empíricos similar.

Por todo ello, se plantearan diferentes estudios de: i) análisis descriptivos y de resultados de detección de situación sobre los SSEV y MMAC deseadas y desarrolladas y de validación de algún instrumento utilizado; ii) patrones psicológicos y educativos en relación en relación con las variables de uso de los SSEV y MMAC; iii) implementación de estrategias de promoción y mejora de SSEV y MMAC en los profesores y iv) implementación de estrategias transversales de promoción y mejora en estudiantes (ej., MOOCs literacy across disciplines), ilustrado por el paso de aprender a escribir a escribir para aprendery de aprender a leer a leer para aprender, con un enfoque del tipo literacy across disciplines, across content, o self-regulated learning (García, 2014; Klein \& Boscolo, 2016; Koster et al., 2015; Li et al., 2018; Scott et al., 2018; Xie et al., 2018; Zen et al., 2019).

\section{REFERENCIAS BIBLIOGRÁFICAS}

De Castro Lozano, C. (2012). El futuro de las tecnologías digitales aplicadas al aprendizaje de personas con necesidades educativas especiales. RED. Revista de Educación a Distancia, 32, 1-43. Retrieved from http://www.redalyc.org/articulo.oa ?id=54724591007

Álvarez, M. L., \& García, J. N. (2014). Evolution of the writing process from Primary to Secondary Education. Journal of Psychodidactics, 19(1), 5-26. doi: 10.1387/RevPsicodidact.6518.

Álvarez, M. L., \& García, J. N. (2015). The orchestration of processes in relation with the product, and the role of psychological variables in writing composition. Anales de Psicología (English Edition), 30(2), 1-24. doi: 10.6018/analesps.31.1.169621

Améstica, L. R., Llinas-Audet, X., \& Sánchez, I. R. (2014). Retorno de la Educación Superior en Chile: efecto en la movilidad social a través del estimador de diferencias en diferencias. Formación Universitaria, 7(3), 23-32. doi: 10.4067/S0718-50062014000 300004

Arias-Gómez, D. H., Díaz-Flórez, 0. C., Garzón-Barragán, I., León-Palencia, A. C., Rodríguez-Ávila, S. P., \& Valbuena-Ussa, E. O. (2018). Entre las exigencias de calidad y las condiciones de desigualdad: Formación inicial de profesores en Colombia. Bogotá: UPN

Barnet, R. (2001). Los límites de la competencia. El conocimiento, la educación superior y la sociedad. Barcelona: Gedisa

Barrio, J. A., \& Barrio, A. (2018). Análisis de la habilidad comunicativa docente y pautas de actuación. Atraer la atención hablando: Un reto para la enseñanza universitaria. International Journal of Developmental and Educational Psychology, 30, 1(1), 73-84.

Batista-Foguet, J. M., Coenders, G., \& Alonso, J. (2004). Análisis factorial confirmatorio. Su utilidad en la validación de cuestionarios relacionados con la salud. Medicina Clínica, 122(1), 21-27. 
Canedo-García, A., García-Sánchez J. N., \& Pacheco-Sanz D. I. (2017). A systematic review of the effectiveness of intergenerational programs. Frontiers in Psychology, 8(1882), 1-13. doi: 10.3389/fpsyg.2017.01882

Cantoral, R. (2014). Teoría Socioepistemológica de la Matemática Educativa. Estudios sobre construcción social del conocimiento. Barcelona: Gedisa

CEEDAR (2019a). Evidences Based Practices. Ceedar innovation configurations. Tools for programs reforms. Retrieved from http://ceedar.education.ufl.edu/tools/innovation-configurations/

CEEDAR (2019b). The CEEDAR Center Evidence Standards. Retrieved form http://ceedar.education.ufl.edu/wp-content/uploads/2014/08/Evidence-Based-Practices-guide.pdf

Chen, Y. H., \& Lin, Y. J. (2018). Validation of the Short Self-Regulation Questionnaire for Taiwanese College Students (TSSRQ). Frontiers in Psychology, 9(259), 1-10. doi: 10.3389/fpsyg.2018.00259

CUC (2018). Autoinforme de Acreditación. Barranquilla: CUC

Cuesta M., Suárez-Álvarez J., Lozano L. M., García-Cueto E., \& Muñiz J. (2018) Assessment of Eight Entrepreneurial Personality Dimensions: Validity Evidence of the BEPE Battery. Frontiers in Psychology, 9(2352), 1-10. doi: 10.3389/fpsyg.2018.02352

De Castro Lozano, C. (2012). El futuro de las tecnologías digitales aplicadas al aprendizaje de personas con necesidades educativas especiales. Revista de Educación a Distancia, 32, 1-43. Retrieved from http://www.redalyc.org/art iculo.oa ?id=54724591007

Díaz, C., \& García, J. N. (2016). Identification of relevant elements for promoting eficient interventions in older people. Journal of Psychodidactics, 21(1), 157-173. doi: 10.1387/RevPsicodidact.13854

Díaz-Flórez, 0. C. (2018). Las competencias en la educación superior. Debates contemporáneos. Tesis Doctoral. Bogotá: UPN

Fidalgo, R., \& García, J. N. (2008). Las directrices del Espacio Europeo de Educación Superior en el marco legislativo del Sistema Universitario Español. Aula Abierta, 35 (1, 2), 35-48.

García, J. N. (2002). El aprendizaje basado en problemas. Ilustración de un modelo de aplicaciones en Psicopedagogía. Cultura y Educación, 14(1), 1-15.

García, J. N. (Ed.) (2014). Prevención en Dificultades del Desarrollo y del Aprendizaje. Instrumentos y programas de actuación en DDA. Madrid: Pirámide. (Digital CD).

García, J. N., Pacheco, D. I., Díaz, C., \& Canedo, A. (2016). Fidelidad de las prácticas basadas empíricamente en dificultades del desarrollo y del aprendizaje. En J. L. Castejón Costa (Coord.) Psicología y Educación: Presente y Futuro (pp. 75-91). Alicante: ACIPE. Retrieved from: http://www.cipe2016.com/cipe_fin al_capitulos.pdf

García, R. (2011). Interdisciplinariedad y sistemas complejos. Revista Latinoamericana de metodología de las ciencias Sociales, 1(1), 66-101.

García-Martín, J., \& García, J. N. (2013). Patterns of Web 2.0 tool use among young Spanish people. Computers and Education, 67, 105-120, doi: 10.1016/j.compedu. 2013.03.003

García-Martín, J., \& García-Sánchez, J. N. (2015). Use of Facebook, Tuenti, Twitter and Myspace among young Spanish people. Behaviour \& Information Technology, 34(7), 685-703. doi: 10.1080/0144929X.2014.993428

García-Martín, J., \& García-Sánchez, J. N. (2017). Pre-service teachers' perceptions of the competence dimensions of digital literacy and of psychological and educational measures. Computers \& Education, 106, 1-14. doi: 10.1016/j.compedu. 2016.12.010

García-Martín, J., \& García-Sánchez, J. N. (2018). The instructional effectiveness of two virtual approaches: processes and product. Journal of Psychodidactics, 23(2), 117-127. doi: 


\subsection{6/j.psicod.2018.02.002}

García-Martín, J., García-Sánchez, J. N., Álvarez-Fernández, M. L., \& Diez Caso, H. (2014). Mejoras en la competencia digital tras la aplicación de un programa de competencias ocupacionales [Improvements in digital competence after the implementation of an occupational competencies program]. European Journal of Education and Psychology, 7(2), 73-81. doi: 10.1989/ejep.v7i2.180

Gil, M. D., Cecato, R., \& Marí, M. I. (2018). Validación y adaptación española de la escala Shell de evaluación del inicio de la escritura en 5 y 5 años. Revista de Psicología Clínica con Niños y Adolescentes, 5(2), 55-60.

González, L., \& González, M. R. (2014). Evaluación de pares y coevaluación en estudiantes y docentes universitarios: una experiencia formativa para impulsar el modelo educativo. International Journal of Developmental and Educational Psychology, 2(1), 501-508.

Habók, A., \& Magyar, A. (2018). Validation of a self-regulated foreign language learning strategy questionnaire through multidimensional modelling. Frontiers in Psychology, 9, 1-11. doi: 10.3389/fpsyg.2018.01388

Karagiannopoulou, E., Milienos, F. S., \& Athanasopoulos, V. (2018). Associations between defense stylesstyles, approaches to learning, and achievement among University Students. Frontiers in Education, 3(53), 1-10. doi: 10.3389/feduc.2018. 00053

Klein, P. D., \& Boscolo, P. (2015). Trends in research on writing as a learning activity. Journal of Writing Research, 7(3), 311-350. doi: 10.17239/jowr-2016.07.03.01

Koster, M., Tribushinina, E., de Jong, P. F., \& van den Bergh, H. (2015). Teaching children to write: a meta-analysis of writing intervention research. Journal of Writing Research, 7(2), 249-274. doi: 10.17239/jowr-2015.07.02.2

Li, J., Ye, H., Tang, Y., Zhou, Z., \& Hu, X. (2018). What are the effects of self-regulation phases and strategies for Chinese students? A meta-analysis of two decades research of the association between self-regulation and academic performance. Frontiers in Psychology, 9, 1-13. doi: 10.3389/fpsyg.2018.02434

Marin-González, F. V., Niebles Lezama, M., Sarmiento, M., \& Valbuena Duarte, S. (2017). Mediación de las tecnologías de la información en la comprensión lectora para la resolución de problemas aritméticos de enunciado verbal. Espacios, 38(20), 1-20. Retrieved from http://www.revistaespacios.com/a17v38n20/a17v38n20p20.pdf

Marín-González, F. V., Cabas, L., \& Paredes-Chacín, A. (2018). Formación integral en profesionales de la ingeniería. Análisis en el plano de la calidad educativa. Formación universitaria, 11(1),1324. doi: $10.4067 / S 0718-50062018000100013$

Martínez-Ferrer, B., Moreno, D., \& Musitu, G. (2018). Are adolescents engaged in the problematic use of social networking sites more involved in peer aggression and victimization? Frontiers in Psychology, 9(801), 1-13. doi: 10.3389/fpsyg.2018.00801

OCDE (2016). La educación en Colombia. MEC: OCDE. https://www.mineducacion. gov.co/1759/articles-356787_recurso_1.pdf

Oriol, X., Miranda, R., Oyanedel, J. C., \& Torres, J. (2017). The role of self-control and grit in domains of school success in students of Primary and Secondary School. Frontiers in Psychology, 8(1716), 1-9. doi: 10.3389/fpsyg.2017.01716

Padrón, J. (1998). La estructura de los procesos de investigación., Revista Educación y Ciencias Humanas, 9(17), 33-45.

Pérez, E. R., \& Medrano, L. (2010). Análisis factorial exploratorio: bases conceptuales y metodológicas. Revista Argentina de Ciencias del Comportamiento, 2(1), 58-66. Retrieved from 
http://www.redalyc.org/ html/3334/333427068006/

Peris, M., Maganto, C., \& Garaigordobil, M. (2018). Escala de riesgo de adicción-adolescente a las redes sociales e internet: fidelidad u validez (ERA-RSI). Revista de Psicología Clínica con Niños y Adolescentes, 5(2), 30-36.

Puente-Martínez, A., Páez, D., Ubillos-Landa, S., \& Da Costa-Dutra, S. (2018). Examining the structure of negative affect regulation and its association with hedonic and psychological wellbeing. Frontiers in Psychology, 9(1592), 1-14. doi: 10.3389/fpsyg. 2018.01592

Ravitch, D. (2013). Reign of error: the hoax of the privatization movement and the danger to America's Public Schools. New York: Alfred A. Knopf.

Rey, M., Mnez, M. R., \& Calonge, I. (2018). Adaptación española de la Children's Hassles Scale: Escala de estresores cotidianos en la infancia. Revista de Psicología Clínica con Niños y Adolescentes, 5(2), 9-16.

Robledo, P., \& García, J. N. (2018). Description and analysis of strategy-focused instructional models for writing. En R. Fidalgo y T. Olive (Series Eds.) y R. Fidalgo, K. R. Harris, y M. Braaksma, (Eds.), Studies in Writing Series: Design Principles for Teaching Effective Writing (pp. 38-65). Leiden: Brill.

Romera, E. M., Herrera, M., Casas, J. A., Ortega, R., \& del Rey, R. (2018). How much do adolescents cybergossip? Scale development and validation in Spain and Colombia. Frontiers in Psychology, 9(126), 1-10. doi: 10.3389/fpsyg.2018.00126

Scott, C. E., McTigue, E. M., Miller, D. M., \& Washburn, E. K. (2018). The what, when and how of preservice teachers and literacy across the disciplines: A systematic literature review of nearly 50 years of research. Teaching and Teacher Education 73, 1-13. doi: 10.1016/j.tate.2018.03.010

Sinval, J., Marques-Pinto, A., Queirós, C., \& Marôco, J. (2018) Work engagement among rescue workers: psychometric properties of the Portuguese UWES. Frontiers in Psychology, 8(2229), 1-16. doi: 10.3389/fpsyg.2017.02229

Sunkel, G., Trucco, D., \& Espejo, A. (2013). La integración de las tecnologías digitales en las escuelas de América Latina y el Caribe: una mirada multidimensional. Santiago de Chile: CEPAL.

Taasoobshirazi, G., \& Wang, S. (2016). The performance of the SRMR, RMSEA, CFI, and TLI: An examination of simple size, path size, and degrees of freedom. Journal of Applied Quantitative Methods, 11(3), 31-39.

Tamayo, L. A., Niño, L. S., Cardozo, L. S., \& Bejarano, 0. (2018). ¿Hacia dónde va la evaluación? Aportes conceptuales para pensar y transformar las prácticas de evaluación. Bogotá: IDEP

USOE (2019). OSEP Ideas that Work. Office of Special Education Programs, U.S. Department of Education. Retrieved from https://www.osepideasthatwork.org/

Ventura-León, J. L. (2018). ¿Existen los instrumentos validos? Un debate necesario. Gaceta Sanitaria, 31(1), 71-71. doi:10.1016/j.gaceta.2016.09.007

Ventura-León, J. L., Barboza-Palomino, M., \& Caycho, T. (2018). ¿Son necesarios los instrumentos equitativos? Educación Médica, 126-127. doi: 10.1016/j.edumed. 2017.03 .022

Ventura-León, J. L., \& Caycho, T. (2017). El coeficiente omega: un método alternativo para la estimación de la confiabilidad. Revista Latinoamericana de Ciencias Sociales, Niñez y Juventud, 15(1), 625-627. Retrieved from: http://www.redalyc.org/articulo. oa?id=77349627039

Ventura, J. L., Caycho, T., Vargas, D., \& Flóres, G. (2018). Adaptación y validación de la Escala de Tolerancia a la Frustración (ETF) en niños peruanos. Revista de Psicología Clínica con Niños y Adolescentes, 5(2), 23-29.

Widlund, A., Tuominen, H., \& Korhonen, J. (2018). Academic well-being, mathematics performance, and educational aspirations in Lower Secondary Education: changes within a school year. 


\section{PERSPECTIVAS TEÓRICAS}

Frontiers in Psychology, 9(297), 1-20. doi: 10.3389/ fpsyg.2018. 00297

Xie, C., Wang, M., \& Hu, H. (2018). Effects of constructivist and transmission instructional models on mathematics achievement in Mainland China: A meta-analysis. Frontiers in Psychology, 9(1923), 1-18. doi: 10.3389/fpsyg.2018.01923

Zeng, G., Chen, X., Cheung, H. Y., \& Peng, K. (2019). Teachers' growth mindset and work engagement in the Chinese educational context: well-being and perseverance of effort as mediators. Frontiers in Psychology, 10(839), 1-10. doi: 10.3389/fpsyg.20 19.00839 